\title{
Preparation and Characterization of Polyvinyl Butyral Nanofibers Containing Silver Nanoparticles
}

\author{
Fatma Yalcinkaya*, Baturalp Yalcinkaya, Jiri Maryska \\ Department of Nanotechnology and Informatics, Centre for Nanomaterials, Advanced Technologies and \\ Innovation, Technical University of Liberec, Studentska 2, 46117, Liberec, Czech Republic \\ Email: "yenertex@hotmail.com
}

Received 5 November 2015; accepted 5 January 2016; published 11 January 2016

\begin{abstract}
Various concentrations of $\mathrm{AgNO}_{3}$ added polymer solutions were electrospun using needleless electrospinning system. Silver nanoparticles were prepared by reducing silver salt using ascorbic acid which is a mild reducing agent. Antibacterial activity of samples tested. Results showed that with the same amount of Silver NPs, higher concentration of PVB polymer solution has better efficiency against to $E$. coli owing to higher productivity.
\end{abstract}

\section{Keywords}

Ag, PVB, Nanofiber, Antibacterial, Filtration

\section{Introduction}

Rapid worldwide population growth, climate change and industrialization is going to lead to an extensive environmental pollution. Increasing pollution reduces clean water resources and demand of clean water increasing day by day. Production of stable, cost and energy efficient material is the need of the water industry.

Nanomaterials may provide solutions to environmental problems in the fields of water treatment. Nanofibers surface/volume ratio and morphology of nanoparticles (NPs) endow contribute them with unique physical and chemical properties compared to macroscopic size materials. Application of nanofiber with NPs has attracted extensive research as memory device [1], drug delivery and tissue engineering [2], membrane separators for lithium ion batteries [3], solar cells [4] [5], waste water treatment [6], biosensors [7].

Polyvinylbutyral (PVB) polymers have been extensively used for many applications, since PVB is a low-cost alternative that offers strong binding ability, flexibility, optical clarity, and adhesion to many surfaces [8]. Strong fiber structure and low-cost alternative makes PVB nanofibers good candidate for wastewater treatment. Silver nitrate NPs were used to enhance efficiency of nanofibers.

Silver nitrate $\left(\mathrm{AgNO}_{3}\right)$ is a colorless (white) inorganic compound to produce silver nanoparticles. Many researchers have been evaluating the ability of the silver ion at inactivating Escherichia coli, a microorganism

\footnotetext{
${ }^{*}$ Corresponding author.
}

How to cite this paper: Yalcinkaya, F., Yalcinkaya, B. and Maryska, J. (2016) Preparation and Characterization of Polyvinyl Butyral Nanofibers Containing Silver Nanoparticles. Journal of Materials Science and Chemical Engineering, 4, 8-12. 
commonly used as an indicator for fecal contamination and as a surrogate for pathogens in drinking water treatment [9]. $\mathrm{AgNO}_{3}$ dissolves in many solvents include acetic acid and water (1).

$$
\mathrm{AgNO}_{3} \text { (solid) }+\mathrm{H}_{2} \mathrm{O} \text { (liquid) } \rightarrow \mathrm{AgNO}_{3} \text { (aqua) }
$$

A combination of PVB nanofibers and Ag NPs were obtained by electrospinning polymer solutions containing $\mathrm{AgNO}_{3}$ salts, which were then reduced into particles in electrospun polymer nanofibers. Figure $\mathbf{1}$ is a schematic representing the procedure for the electrospinning of Ag NPs-polymer nanofiber composites.

In this study, PVB nanofibers with Ag NPs were fabricated. PVB solution was mixed with $\mathrm{AgNO}_{3}$ salt and then spun to form nanofiber web. The nanofiber web was immersed into ascorbic acid for reduction of Ag.

Nanofibers and NPs might be used in future at industrial scale water purification. In future, combination of both might be the best option for waste water treatment.

\section{Material and Methods}

\subsection{Preparation of Nanofiber Web}

PVB was purchased from Kuraray (grade Mowital B $60 \mathrm{H}$; Germany) with a molecular weight of $60.000 \mathrm{~g} / \mathrm{mol}$. Acetic acid 99.8\% (Pentas.r.o., Czech Republic) and distilled water was used as solvent. Silver nitrate salt was purchased from Lach-Ners.r.o. (Czech Republic) and ascorbic acid (Pentas.r.o., Czech Republic) was used for reduction of Ag.

6 and $10 \mathrm{wt} \%$ of PVB solution were prepared in the mixture of acetic acid and distilled water. $4 \mathrm{wt} \%$ distilled water was used for both solution system. It was observed that adding water into acetic acid increase the conductivity of solvent. The same solutions were prepared by adding $2 \mathrm{wt} \% \mathrm{AgNO}_{3}$.

All the solutions were spun at the same conditions using rod electrospinning system as shown in Figure 2. In the rod electrospinning system, a metal rod $10 \mathrm{~mm}$ in diameter linked with a high voltage supply is the spinning electrode. The collector is grounded. A droplet of polymer solution is placed at the upper end of the rod. Jets develop on the surface of the droplet, mainly close to its periphery.

Images of the microstructure of the nanofiber membrane were taken by scanning electron microscope (SEM; Tescan Vega3 SB). Image-J software was used to determine the fibre diameter and diameter distribution.

\subsection{Synthesis of Ag-NPs by Ascorbic Acid}

Silver nanoparticles can be prepared by milder reducing agent like ascorbic acid. This is the slower growth process. Reduction of Ag+ occurs on silver seeds [10]. Fabricated nanofibers were immersed into ascorbic ac$\mathrm{id} /$ water solution with the $1 / 3 \mathrm{wt} \%$ ratio. Samples were kept 5 minutes in reduction agent and then dried at room temperature. The color change was observed. Resultant silver lustre and brown color nanofibers were prepared for the antibacterial test.

\subsection{Antibacterial Test}

Bacteria Escherichia coli (E. coli) were purchased from Czech Collection of Microorganisms, Masaryk University. Incubation of bacteria was performed on a sterile agar with broth agar medium from OxoidCz Company. Antimicrobial activities of complexes were determined according to ASTM E 2149-01 and AATCC test method

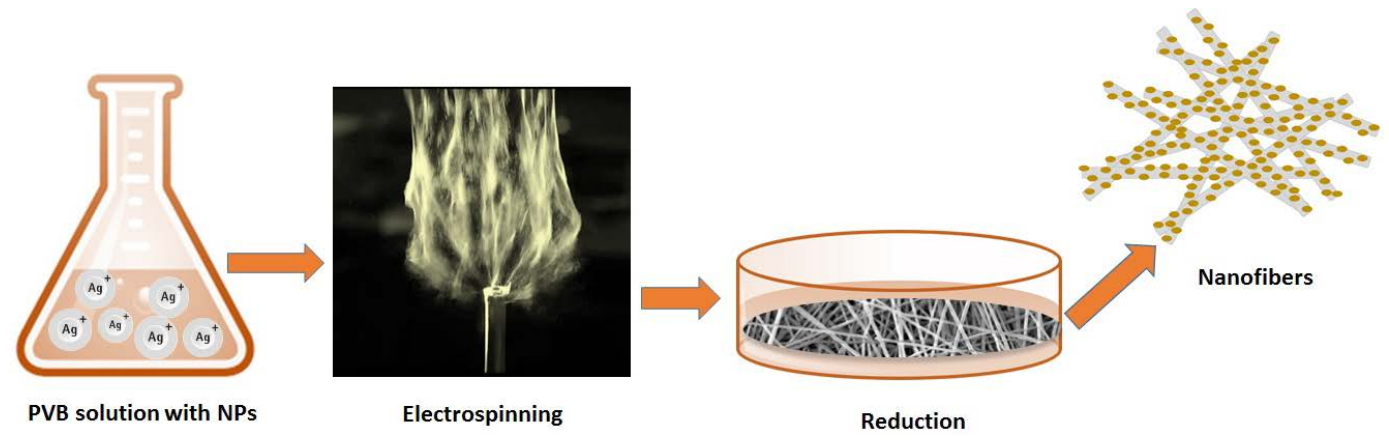

Figure 1. Schematic of fabricating Ag NPs-polymer nanofiber composites via rod electrospinning. 


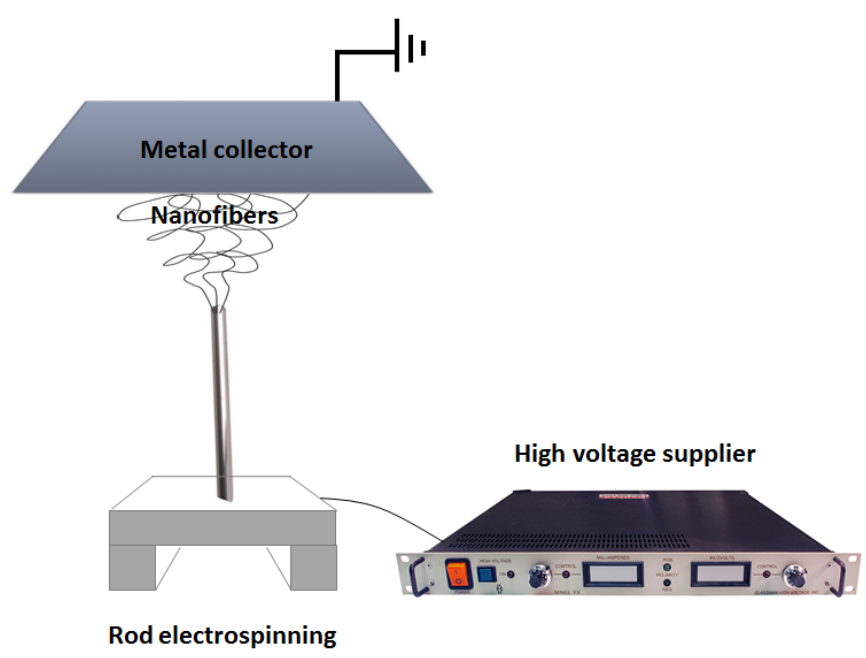

Figure 2. Schematic of rod electrospinning.

100 (Standard test method for determining the antimicrobial activity of immobilized antimicrobial agents under dynamic contact conditions).

Samples were cut into $4.8 \times 4.8 \mathrm{~cm}^{2}$. The samples were sterilized in an oven at $70^{\circ} \mathrm{C}$ for $60 \mathrm{~min} .6$ and $10 \mathrm{wt} \%$ PVB solution without $\mathrm{AgNO}_{3}$ were prepared as blank samples. The antimicrobial test against Gram-negative (E. coli) was performed. The microorganisms were cultivated in sterilized LB broth medium and then incubated overnight at $37^{\circ} \mathrm{C}$ within a shaking incubator. Colony forming units (CFU) was arranged as $10^{3}$ for this test.

Sterilized nanofiber webs were placed into a sterilized test tube and inoculated with $30 \mathrm{ml}$ of $E$. coli bacterial suspension. In " 0 " contact time and after 1, 2, 3 and 4 hours, $600 \mathrm{~mL}$ of bacterial suspension was extracted and quickly spread on Tryptic Soy agar plates. The number of viable E. coli was determined by plating the extracted solution onto the Tryptic Soy agar plates and counting colonies after 24 hours of incubation at $37^{\circ} \mathrm{C}$.

The percentage reduction of test microorganisms in test tubes with nanofiber membranes after 24 hours was calculated using the "Equation (2)".

$$
\mathrm{R} \%=\left(\mathrm{A}_{0}-\mathrm{A}_{1}\right) / \mathrm{A}_{0} * 100(2)
$$

where $\mathrm{R}$ is the reduction of test microorganism in percentage; $\mathrm{A}_{1}$ is the number of bacteria recovered from the inoculated nanofiber membrane with the nanoparticles in the test tube after specified contact time, and $\mathrm{A}_{0}$ is the number of bacteria recovered from the inoculated nanofiber membrane without the nanoparticles in the test tube at "0" contact time.

\section{Result and Discussion}

\subsection{Surface Morphology}

The SEM images of PVB nanofibers by adding Ag-NPs was taken and shown as in Figure 3.

Figure 3 shows that $6 \%$ wt PVB has beads structure on the surface due to lower concentration and viscosity. It is well known from literature that increasing concentration of solution eliminate bead structure and increase fiber diameter.

\subsection{Antibacterial Efficiency}

The antibacterial property of silver has been known very well. Bacteria needs and uses enzymes to metabolize oxygen, however, Ag ions damage the enzyme and block the oxygen take up. Antibacterial properties of PVB nanofiber membranes with nanoparticles were analyzed. Gram negative (E. coli) bacteria was chosen. Figure 4 shows kinetic effects of the silver NPs on E. coli growth curves.

Figure 4 shows the reduction of test microorganisms $\mathrm{R}$ caused by adding $\mathrm{AgNO}_{3}$ salt entrapped in PVB nanofiber membranes. Within less than $4 \mathrm{~h}$, Ag nanoparticles with $10 \mathrm{wt} \%$ PVB completely eradicated bacteria in cultures, whereas 6 wt\% PVB with Ag-NPs reduced viability of E. coli by 87, respectively, after 4 h of treatment. 

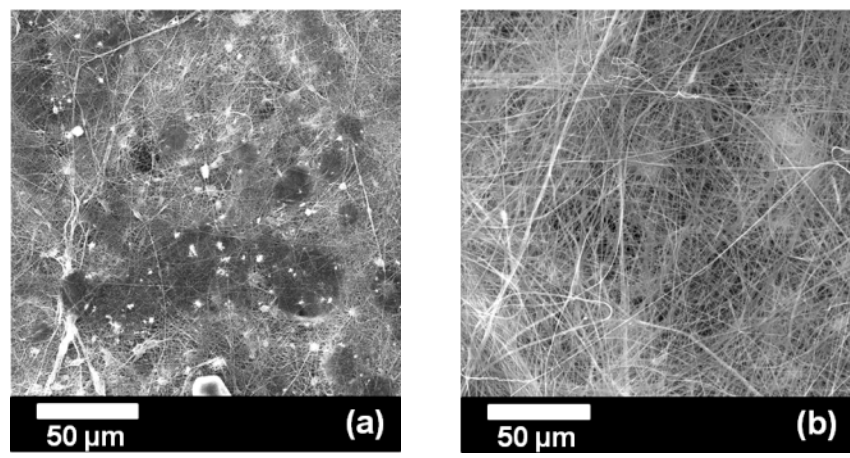

Figure 3. SEM image of (a) 6\% PVB+Ag-NPs; (b) 10\% PVB + Ag-NPs.

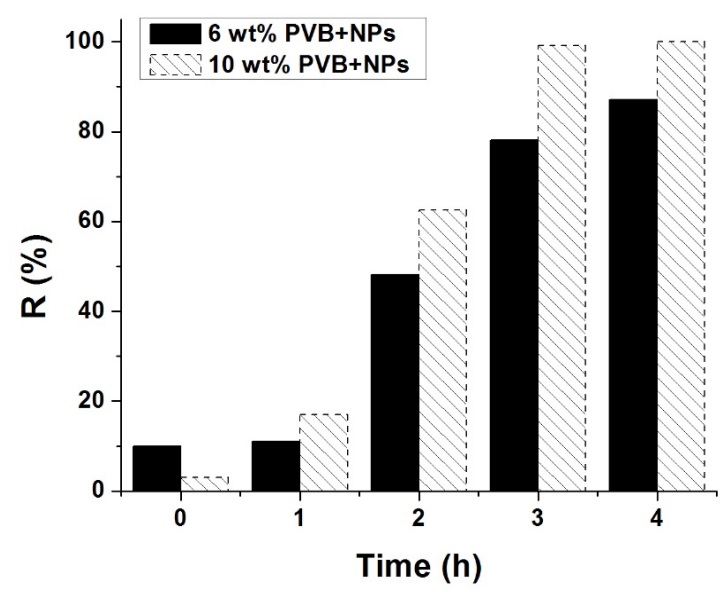

Figure 4. Antibacterial activity of Ag-entrapped PVB nanofibers.

In both cases of tested microorganisms, number of bacteria recovered from the inoculated nanofiber membrane with the nanoparticles in the test tube after 4 hours was reduced. However, the reductions of tested microorganisms $\mathrm{R}$ were different and depended on the amount of nanoparticles in nanofiber membranes. It was observed that productivity of $10 \mathrm{wt} \%$ PVB solution is higher than 6 which causes higher amount of material. It seems that by using $10 \mathrm{wt} \% \mathrm{PVB}$, productivity improved and amount of NPs per $\mathrm{cm}^{2}$ was increased. It is visible from SEM image that the surface fiber structure of $10 \mathrm{wt} \%$ PVB is smoother than $6 \mathrm{wt} \%$ PVB nanofibers. We may, therefore, suggest that antibacterial activity of Ag-NPs against E. coli is related to different membrane layer thickness and structure.

These results confirmed that nanofiber membranes with incorporated Ag-NPs enhanced antimicrobial activity against bacteria $E$. coli compared to the pure PVB nanofibers without any nanoparticles.

\section{Conclusions}

The present study demonstrates an economical and environmentally affable approach for the antibacterial silver NPs entrapped in nanofibers were prepared by reduction of silver salt. The PVB nanofibers containing Ag NPs were prepared by rod electrospinning. The nano webs were characterized by SEM and antibacterial efficiency was evaluated. SEM images showed that $6 \mathrm{wt} \%$ PVB + Ag-NPs has beads structure, whereas $10 \mathrm{wt} \%$ has beads free and smooth fibers. It was observed that $10 \mathrm{wt} \%$ PVB solution has higher productivity than $6 \mathrm{wt} \%$ solution. Antibacterial test demonstrated that $10 \mathrm{wt} \% \mathrm{PVB}+$ Ag-NPs solution has very good antibacterial efficiency. After 4 hours of contact time, it was observed that $10 \mathrm{wt} \%$ PVB + Ag-NPs has almost 100\% reduction of bacteria.

Nanofibers itself are good candidate for water filtration due to high specific surface area and small pore size. Ag-NPs entrapped nanofibers produced by needleless electrospinning might be good candidate for wastewater treatment. 


\section{Acknowledgements}

The results of this project LO1201 were obtained through the financial support of the Ministry of Education, Youth and Sports in the framework of the targeted support of the "National Programme for Sustainability I".

\section{References}

[1] Tseng, R.J., Huang, J., Ouyang, J., Kaner, R.B. and Yang, Y. (2005) Polyaniline Nanofiber/Gold Nanoparticle Nonvolatile Memory. Nanoletters, 5, 1077-1080. http://dx.doi.org/10.1021/nl0505871

[2] Sridhar, R., Lakshminarayanan, R., Madhaiyan, K., Barathi, V.A., Lim, K.H. and Ramakrishna, S. (2015) Electrosprayed Nanoparticles and Electrospun Nanofibers Based on Natural Materials: Applications in Tissue Regeneration, Drug Delivery and Pharmaceuticals. Chem. Soc. Rev., 44, 790-814. http://dx.doi.org/10.1039/C4CS00226A

[3] Yanilmaz, M., Lu, Y., Dirican, M., Fu, K. and Zhang, X. (2014) Nanoparticle-on-Nanofiber Hybrid Membrane Separators for Lithium-Ion Batteries via Combining Electrospraying and Electrospinning Techniques. Journal of Membrane Science, 456, 57-65. http://dx.doi.org/10.1016/j.memsci.2014.01.022

[4] Li, J., Chen, X., Ai, N., Hao, J., Chen, Q., Strauf, S. and Shi, Y. (2011) Silver Nanoparticle Doped TiO 2 Nanofiber Dye Sensitized Solar Cells. Chemical Physics Letters, 514, 3141-3145. http://dx.doi.org/10.1016/j.cplett.2011.08.048

[5] Lee, J.H., Ahn, K., Kim, S.H., Jeong, S.Y., Bae, J.S., Hong, T.E., Kim, H.G. and Cho, C.R. (2015) Efficiencies of Dye-Sensitized Solar Cells with Hollow $\mathrm{SnO}_{2}$ Nanofiber/TiO ${ }_{2}$ Nanoparticle Composite Photoanodes. J Nanosci Nanotechnol, 15, 244-247. http://dx.doi.org/10.1166/jnn.2015.8399

[6] Qu, X., Alvarez, P.J.J. and Li, Q. (2013) Applications of Nanotechnology in Water and Wastewater Treatment. Water Research, 47, 3931-3946. http://dx.doi.org/10.1016/j.watres.2012.09.058

[7] Loaiza, O.A., Lamas-Ardisana, P.J., Añorga, L., Jubete, E., Ruiz, V., Borghei, M., Cabañero, G. and Grande, H.J. (2015) Graphitized Carbon Nanofiber-Pt Nanoparticle Hybrids as Sensitive Tool for Preparation of Screen Printing Biosensors. Detection of Lactate in Wines and Ciders. Bioelectrochemistry, 101, 58-65. http://dx.doi.org/10.1016/j.bioelechem.2014.07.005

[8] Yener, F. and Jirsak, O. (2012) Comparison between the Needle and Roller Electrospinning of Polyvinylbutyral. Journal of Nanomaterials, 2012, 6 p.

[9] http://organobiotech.com/silvernitrate.html

[10] Rashid, M.U., Bhuiyan, Md.K.H. and Quayum, M.E. (2013) Synthesis of Silver Nano Particles (Ag-NPs) and Their Uses for Quantitative Analysis of Vitamin C Tablets. Dhaka Univ. J. Pharm. Sci., 12, 29-33. http://dx.doi.org/10.3329/dujps.v12i1.16297 